\title{
Propriedades do Conjunto de Cantor
}

\section{Cantor Set and its properties}

\author{
Bryan Douglas Nunes Assunção \\ Universidade Federal de Uberlândia (UFU), Instituto de Física, Graduação em Física de Materiais, \\ Uberlândia, MG, Brasil \\ https://orcid.org/0000-0002-4715-3392, bryan.assuncao@ufu.br
}

Fábio José Bertoloto

Universidade Federal de Uberlândia (UFU), Faculdade de Matemática, Uberlândia, MG, Brasil https://orcid.org/0000-0002-2212-2602, bertoloto@ufu.br

\section{Informações do Artigo}

\section{Como citar este artigo}

ASSUNÇÃO, Bryan Douglas Nunes; BERTOLOTO, Fábio José.

Propriedades do Conjunto de Cantor. REMAT: Revista Eletrônica da Matemática, Bento Gonçalves, RS, v. 7, n. 1, p. e3011, 20 de abril de 2021. DOI: https://doi.org/10.35819/ remat2021v7i1id4394

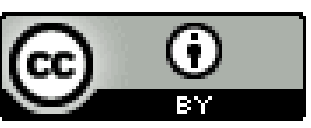

\section{Resumo}

Este trabalho tem por intenção divulgar o já conhecido Conjunto de Cantor. A ideia é exibir uma demonstração mais detalhada de algumas propriedades importantes que ele possui, não sendo, de certa forma, tão comum encontrá-las em textos em português. Também veremos que, a menos de homeomorfismo, o Conjunto de Cantor é o único, como espaço métrico, com todas as propriedades indicadas.

\section{Histórico do Artigo}

Submissão: 5 de agosto de 2020.

Aceite: 8 de fevereiro de 2021 .

\section{Palavras-chave}

Conjunto de Cantor

Enumerabilidade

Conjuntos Homeomorfos

Noções Topológicas na Reta

\section{Keywords}

Cantor Set

Countable Sets

Homeomorphic Sets

Topolical Notions on the Real Line

\begin{abstract}
This work intends to publicize the already well-known Cantor set. The idea is to show a more detailed demonstration of some important properties that it has, in a certain way being not very common to find in texts in Portuguese. We will also see that, except for homeomorphism, the Cantor Set is the only one, as metric space, with all the indicated properties.
\end{abstract}




\section{Introdução}

Este trabalho possui como objetivo contemplar e demonstrar propriedades do Conjunto de Cantor. Filho de dinamarqueses, mas nascido na Rússia, Georg Cantor (1845-1918) doutorou-se em matemática pela Universidade de Berlim. Ficou conhecido por ser um dos criadores do Congresso Internacional de Matemáticos (ICM) ao lado de Felix Klein, e por ter elaborado a moderna Teoria dos Conjuntos, que abriu as portas para ampliar as fronteiras da Matemática e definir o conceito de infinito.

Seus trabalhos geraram embates e polêmicas entre os matemáticos da época. Tempos depois, tais trabalhos foram reconhecidos, levando-o a ser nomeado membro honorário da Lodon Mathematical Society e homenageado com a medalha de Royal Society London.

David Hilbert (1862-1943), um de seus poucos defensores na época, afirmou: "Que ninguém seja capaz de nos tirar do paraíso que Cantor criou para nós”. Reconhecidamente religioso, Cantor era visto com maus olhos pela comunidade matemática e sofreu boicotes e perseguições, o que o deixou perturbado, levando-o a uma internação em uma clínica psiquiátrica na Alemanha, até os seus últimos dias.

Mais detalhes sobre a vida e os trabalhos de Cantor podem ser encontrados nos estudos de Freira (1992) e IMPA (2017). As principais referências para o trabalho são Lima (2018) e Willard (2004).

Este texto inicia com uma seção sobre conceitos preliminares. Na sequência, temos uma seção onde o Conjunto de Cantor é definido e tem suas propriedades mais conhecidas demonstradas. Por fim, vemos que, a menos de homeomorfismo, o Conjunto de Cantor é único com todas as propriedades apresentas, ou seja, é o único espaço métrico compacto não vazio, perfeito e totalmente desconexo. Este resultado é conhecido como o Teorema de Brouwer (BROUWER, 1910).

\section{Preliminares}

Nesta seção, apresentamos algumas definições que são necessárias para uma melhor compreensão do conteúdo. 


\subsection{Espaços métricos}

Para esta seção, utilizamos, em grande parte, Munkres (2000) e Willard (2004).

Definição 2.1. Seja um conjunto $M \neq \emptyset$. Uma métrica no conjunto $M$ é uma função $d: M \times M \longrightarrow \mathbb{R}$ que associa, a cada par ordenado de elementos $(x, y) \in M \times M$, um número real não negativo $d(x, y)$ denominado distância de $x$ a $y$, de modo que sejam satisfeitas as seguintes condições, para quaisquer $x, y, z \in M$ :

(D1) $d(x, x)=0$;

(D2) $x \neq y \Rightarrow d(x, y)>0$;

(D3) $d(x, y)=d(y, x)$;

(D4) $d(x, z) \leqslant d(x, y)+d(y, z)$ (desigualdade triangular).

O par $(M, d)$ é chamado espaço métrico.

Exemplo 2.2. Seja $d: \mathbb{R} \times \mathbb{R} \longrightarrow \mathbb{R}$, dada por $d(x, y)=|x-y|$, a função que define a distância entre dois pontos $x, y \in \mathbb{R}$. O par $(\mathbb{R}, d)$ é um exemplo de espaço métrico. Para $n \in \mathbb{N}, n \geq 2$, qualquer espaço $\mathbb{R}^{n}$, com a métrica do soma, do máximo ou euclidiana, são exemplos de espaços métricos.

\subsection{Espaços topológicos}

Definição 2.3. Uma topologia em um conjunto $X$ é uma coleção $\tau$ de subconjuntos de $X$, chamados conjuntos abertos, satisfazendo:

(T1) Qualquer união de elementos de $\tau$ pertence a $\tau$;

(T2) Qualquer interseção finita de elementos de $\tau$ pertence a $\tau$;

(T3) $\emptyset$ e $X$ pertencem $a \tau$.

Dizemos que $(X, \tau)$ é um espaço topológico. Caso não haja confusão, escreveremos apenas $X$ para espaço topológico no lugar de $(X, \tau)$.

Exemplo 2.4. Qualquer espaço métrico $(M, d)$ é um exemplo de espaço topológico. Exemplos de abertos nos espaços métricos são as bolas abertas. Dados $a \in M$ e $r>0, r \in \mathbb{R}$, a bola aberta de centro a e raio $r$ é conjunto

$$
B(a ; r)=\{x \in M ; d(x ; a)<r\} .
$$


Exemplo 2.5. A topologia discreta é formada por todos os subconjuntos de $X$. A mais simples é a topologia trivial, caótica ou cofinita que tem por elementos apenas $X$ e $\emptyset$.

Definição 2.6. Em um espaço topológico $X$, dizemos que $F$ é fechado se o complementar em $X$ $(A=X-F)$ for aberto.

Exemplo 2.7. Exemplos de conjuntos fechados são as bolas fechadas em espaços métricos, ou seja, conjuntos da forma

$$
\bar{B}(a ; r)=\{x \in M ; d(x ; a) \leq r\}
$$

Definição 2.8. Sejam $X$ e $Y$ espaços topológicos. Uma função $f: X \longrightarrow Y$ é contínua se para cada subconjunto aberto $A$ de $Y$, o conjunto $f^{-1}(A)$ é um subconjunto aberto de $X$.

Observação 2.9. De forma equivalente, podemos escrever essa definição dizendo que $f^{-1}(F)$ é fechado em $X$, para todo $F$ fechado em $Y$.

Definição 2.10. Dados espaços métricos $X$ e $Y$, um homeomorfismo entre este espaços é uma função $f: X \longrightarrow Y$ que seja bijetora, contínua, com inversa contínua.

Exemplo 2.11. Dados o cone $C=\left\{(x, y, z) \in \mathbb{R}^{3} ; x^{2}+y^{2}=z^{2}\right.$ e $\left.z \geq 0\right\}$ e o plano $\mathbb{R}^{2}$, podemos definir um homeomorfismo $g: \mathbb{R}^{2} \longrightarrow C$. De fato, basta definir

$$
g(x, y)=\left(x, y, x^{2}+y^{2}\right)
$$

Nem toda função bijetora contínua tem inversa contínua. Basta considerar $f:[0,2 \pi) \longrightarrow S^{1}$, em que $S^{1}$ é a circunferência de centro $(0,0)$ e raio 1 , dada por $f(t)=(\cos (t)$, sen $(t))$. Claro que $f$ é contínua e bijetora. A inversa não é contínua em $(0,1)$, pois para cada $k \in \mathbb{N}$, sejam $t_{k}=2 \pi-(1 / k)$ e $z_{k}=f\left(t_{k}\right)$. Então $\lim z_{k}=(1,0)=p$, mas não é verdade que $\lim f^{-1}\left(z_{k}\right)=\lim t_{k}$ seja igual a $f^{-1}(p)=0$. Aqui consideramos a métrica euclidiana.

Definição 2.12. Sejam $a \in X$, em que $X$ é um espaço topológico. Dizemos que:

(i) a é ponto interior a $X$, se existe um aberto $A \subset X$ tal que $a \in A$. No caso de $A \subset \mathbb{R}^{n}$, se existe $\epsilon>0$, tal que $B(a ; r) \subset X$. Caracteriza-se como o interior de $X$ (int $(X))$ o conjunto de todos os pontos interiores a $X$. Pode-se mostrar que o int $(X)$ é a união de todos os abertos contidos em $X$.

(ii) a é aderente a $X$, se todo aberto em torno de a intercepta $X$. No caso $X \subset \mathbb{R}^{n}$, se a é o limite de alguma sequência $\left(x_{n}\right)$ de pontos de $X$, ou seja, dado $\epsilon>0$, existe $n_{0} \in \mathbb{N}$ tal que para $n \geq n_{0}$, 


$$
\left\|x_{n}-a\right\|<\epsilon
$$

$O$ fecho de $X(\bar{X})$ é o conjunto de todos os pontos aderentes a $X$. É possível mostrar que um conjunto é fechado se, e somente se, $X=\bar{X}$. É claro que sempre vale $X \subset \bar{X}$. Pode-se provar que $\bar{X}$ é a interseção de todos os fechados que contêm $X$;

(iii) a é ponto de acumulação de $X$ se todo aberto em torno de a contém algum ponto de $X$ diferente do próprio a. No caso $X \subset \mathbb{R}^{n}$, quando para todo $\epsilon>0$, valer que $(B(a ; \epsilon) \backslash\{a\} \cap X) \neq \emptyset$. Diz-se que $a \in X$ é ponto isolado de $X$, se a não for ponto de acumulação;

(iv) Uma cobertura aberta de um espaço topológico $X$ é uma coleção de abertos $\left\{A_{i}, i \in I\right\}$, sendo I um conjunto de índices, de $X$ tais que

$$
X=\bigcup_{i} A_{i} .
$$

Uma subcobertura de uma cobertura é uma subcoleção de abertos que ainda cobre $X$.

Dizemos que $X$ é compacto se toda cobertura admite subcobertura finita. No caso de espaços métricos, pode-se mostrar que todo conjunto compacto é limitado e fechado, ou seja, está contido em alguma bola de raio finito. Não vale a recíproca, como pode ser visto em Mathonline (2020). Porém, há uma equivalência no caso dos espaços euclidianos $\mathbb{R}^{n}$.

\subsubsection{Conexidade}

Definição 2.13. (1) Seja $X$ um espaço topológico. Uma separação de $X$ é um par $U, V$ de subconjuntos de $X$ que sejam abertos, não vazios e disjuntos, tal que $X=U \cup V$. X é conexo se não existe uma tal separação.

(2) Se $x \in X$, o maior subconjunto conexo $C_{x}$ contendo $x$ é chamado de componente conexa de $x$.

Exemplo 2.14. $X \subset \mathbb{R}$ é conexo se, e somente se, $X$ é um intervalo. Ver Lima (2014, p. 56, Teorema 30).

Definição 2.15. Dado um espaço topológico $X$, dizemos que um conjunto $A \subset X$ é:

(i) Totalmente desconexo se, e somente se, as componentes conexas de cada $x \in X$ são pontos. No caso de $X \subset \mathbb{R}$, é o mesmo que dizer que não contém intervalos, ou seja, não contém pontos interiores. 
(ii) Perfeito, se todos os seus pontos são de acumulação.

\subsection{Representações em base 3}

Definição 2.16. Dado $x \in[0,1)$, representar $x$ na base 3 significa escrever $x=\left(0, x_{1}, x_{2}, x_{3}, \cdots, x_{n}, \cdots\right)_{3}$, onde cada um dos dígitos $x_{n}$ são iguais a 0, 1 ou 2, de tal modo que

$$
x=\frac{x_{1}}{3^{1}}+\frac{x_{2}}{3^{2}}+\frac{x_{3}}{3^{3}}+\cdots+\frac{x_{n}}{3^{n}}+\cdots .
$$

Proposição 2.17. Um número da forma $(0,0, \ldots, 2,2, \ldots)_{3}$, com 2 começando na $n+1$-ésima posição, é igual a $(0,0, \ldots, 1,0, \ldots)_{3}$, em que 1 ocupa a $n$-ésima posição.

Prova. Este fato segue diretamente do resultado de somas de progressões geométricas. De fato, utilizando da definição de representação em base 3, obtemos:

$$
\begin{aligned}
(0,0, \ldots, 2,2, \ldots)_{3} & =\sum_{j=n+1}^{\infty} \frac{2}{3^{j}} \\
& =2\left(\sum_{j=0}^{\infty} \frac{1}{3^{j}}-\sum_{j=0}^{n} \frac{1}{3^{j}}\right) \\
& =2\left(\frac{1}{1-\frac{1}{3}}-\frac{1-\left(\frac{1}{3}\right)^{n+1}}{1-\frac{1}{3}}\right) \\
& =\left(\frac{1}{3}\right)^{n} .
\end{aligned}
$$

Proposição 2.18. Se $A=\left\{k 3^{-n+1} ; 0 \leq k \leq 3^{n-1}-1\right.$ e $\left.k \in \mathbb{Z}\right\}$ e $B=\left\{\left(0, a_{1}, \ldots, a_{n-1}\right)_{3} ; a_{1}, \ldots, a_{n-1} \in\right.$ $\{0,1,2\}\}$, então $A=B$.

Prova. Façamos a demonstração em partes.

1. $A \subset B$ : Seja $x \in A$. Então $x=\frac{k}{3^{n-1}}$. Pelo algoritmo da divisão, podemos escrever $k=$ $3 m_{1}+r_{1}$, com $m_{1}, r_{1} \in \mathbb{N}$ e $0 \leq r_{1} \leq 2$. Com isso,

$$
x=\frac{m_{1}}{3^{n-2}}+\frac{r_{1}}{3^{n-1}} .
$$

Prosseguindo, podemos dividir $m_{1}$ por 3 , obtendo $m_{1}=3 m_{2}+r_{2}$, com $m_{2}, r_{2} \in \mathbb{N}$ e $0 \leq r_{2} \leq 2$, resultando em

$$
x=\frac{m_{2}}{3^{n-3}}+\frac{r_{2}}{3^{n-2}}+\frac{r_{1}}{3^{n-1}} .
$$


Prosseguindo com algoritmo da divisão, encontraremos algum $j \in \mathbb{N}$, tal que $m_{j-1}=3 m_{j}+r_{j}$, com $m_{j}, r_{j} \in \mathbb{N}$, sendo $1 \leq m_{j} \leq 2$ e $0 \leq r_{j} \leq 2$. Assim,

$$
x=\frac{m_{j}}{3^{n-(j+1)}}+\frac{r_{j}}{3^{n-j}}+\ldots+\frac{r_{2}}{3^{n-2}}+\frac{r_{1}}{3^{n-1}}=\left(0,0, \ldots, 0, m_{j}, r_{j}, \ldots, r_{2}, r_{1}\right)_{3},
$$

ou seja, $x \in B$, concluindo que $A \subset B$. Destacamos que $m_{j}$ está na posição $n-(j+1)$. Também, $j<n$ deve ocorrer, pois $0 \leq x \leq 1-\frac{1}{3^{n-1}}<1$. Caso $j \geq n$, ocorreria $x>1$.

2. $B \subset A$ : Seja $x \in B$. Então

$$
x=\frac{a_{1}}{3}+\frac{a_{2}}{3^{2}}+\ldots+\frac{a_{n-1}}{3^{n-1}}=\frac{3^{n-2} a_{1}+3^{n-3} a_{2}+\ldots+3 a_{n-2}+a_{n-1}}{3^{n-1}},
$$

com $a_{i} \in\{0,1,2\}, i=1, \ldots, n-1$ e $n \in \mathbb{N}$ tal que $n \geq 2$. Escrevemos $k=3^{n-2} a_{1}+3^{n-3} a_{2}+$ $\ldots+3 a_{n-2}+a_{n-1}$. Claro que $k \in \mathbb{N}$. Agora, de $0 \leq a_{1}, a_{2}, \ldots, a_{n-1} \leq 2$, obtemos que $k \geq 0$ e que

$$
k \leq 2\left(3^{n-2}+3^{n-3}+\ldots+3+1\right)=2 \frac{3^{n-1}-1}{2}=3^{n-1}-1,
$$

concluindo que $B \subset A$.

\section{Conjunto de Cantor}

Nesta seção, construímos o conjunto de Cantor e demonstramos algumas de suas propriedades. Mais explicitamente, vamos mostrar que o conjunto de Cantor é totalmente desconexo, compacto, perfeito e não enumerável.

Vejamos sua construção. Ele é obtido como o complementar de uma reunião de intervalos abertos.

Inicialmente, consideramos o intervalo $[0,1]$. Deste, retiramos o seu terço médio aberto, ou seja, o intervalo $\left(\frac{1}{3}, \frac{2}{3}\right)$. Na sequência, retiramos os terços médios dos intervalos restantes, ou seja, de $\left[0, \frac{1}{3}\right]$ e $\left[\frac{2}{3}, 1\right]$ retiramos, respectivamente, os intervalos $\left(\frac{1}{9}, \frac{2}{9}\right)$ e $\left(\frac{7}{9}, \frac{8}{9}\right)$. llustramos até este passo na Figura 1.

Figura 1 - Fragmentação do intervalo [0,1], até a 2ª etapa da construção do Conjunto de Cantor.

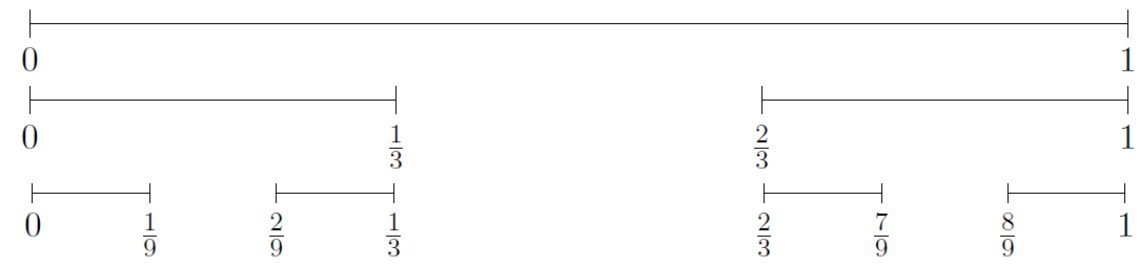

Fonte: Elaboração dos autores (2020). 
Este processo se repete, infinitamente, sempre na etapa seguinte, retirando-se o terço médio aberto dos intervalos que sobraram na etapa anterior.

Notemos que na $n$-ésima etapa, cada intervalo restante, após a retirada, tem comprimento $\frac{1}{3^{n}}$, sendo que há $2^{n}$ destes intervalos.

Denominando por $K$ o conjunto de Cantor e por $C_{n}$ a união dos intervalos restantes na etapa $n \in \mathbb{N}^{*}$, podemos afirmar que

$$
K=\bigcap_{n=1}^{\infty} C_{n}
$$

Em seguida, demonstraremos as quatro propriedades listadas anteriormente.

\section{1 $K$ é totalmente desconexo}

Suponhamos que $K$ possui um interior não vazio e seja $x \in \operatorname{int}(K)$.

Então, devemos ter $\epsilon>0$, tal que $(x-\epsilon, x+\epsilon) \subset K$.

Mas, como existe $n \in \mathbb{N}$, tal que $\frac{1}{3^{n}}<\epsilon$ (comprimento dos intervalos na etapa $n$ ), ocorre que na $n$-ésima etapa não pode estar contido em $K$ um intervalo $(x-\epsilon, x+\epsilon)$, sendo parte dele interceptado pelo complementar de $K$, implicando que o interior deste conjunto é vazio.

\section{2 $K$ é compacto}

Do fato de $K$ estar contido em [0,1], então este é claramente limitado. Como a cada etapa é retirada uma quantidade finita de intervalos abertos e, a quantidade de etapas é enumerável, utilizando Lima (2014, p. 8), podemos afirmar que uma quantidade enumerável de intervalos abertos é retirada de $[0,1]$ para formar o Conjunto de Cantor.

Denominando tais intervalos abertos como $I_{1}, I_{2}, \ldots, I_{n}, \ldots$, teremos que $F=\mathbb{R}-\bigcup_{n=1}^{\infty} I_{n}$ é fechado, pois é o complementar de uma união enumerável de abertos. Isto implica que $K=[0,1] \cap F$ também será fechado. Portanto, $K$ é compacto. 


\section{3 $K$ é perfeito}

Seja $a \in K$. Então, $a$ está sempre em algum intervalo restante de cada etapa da construção do conjunto de Cantor.

Claro que na primeira etapa, $a$ deve estar em $\left[0, \frac{1}{3}\right]$ ou $\left[\frac{2}{3}, 1\right]$.

Denominamos o intervalo, neste caso, ao qual a pertence por $\left[x_{1}, y_{1}\right]$. Na próxima etapa, $a$ estará em algum intervalo da forma $\left[x_{1}, b\right]$ ou $\left[c, y_{1}\right]$, pois foi retirado o terço médio aberto de $\left[x_{1}, y_{1}\right]$. Se estiver no primeiro, denominamos tal intervalo por $\left[x_{2}, y_{2}\right]$, em que $x_{2}=x_{1}$ e $y_{2}=b$ (análogo para o caso do segundo intervalo, em que teríamos $x_{2}=c$ e $y_{2}=y_{1}$ ). $\mathrm{E}$, assim, procedemos indefinidamente, observando que para todo $n \in \mathbb{N}$, sempre ocorre que existem $x_{n}, y_{n} \in K$, com $x_{n} \leq a \leq y_{n}$, em que $y_{n}-x_{n}=\frac{1}{3^{n}}$, conforme já comentado.

Daí, construímos sequências $\left(x_{n}\right)$ e $\left(y_{n}\right)$ de elementos de $K$, onde pelo menos uma delas é não constante e de infinitos termos distintos, $\operatorname{com} \lim x_{n}=\lim y_{n}=a$, ou seja, $a$ é ponto de acumulação.

\section{4 $K$ é não enumerável}

Os elementos de $K$ podem ser escritos na base 3, conforme Definição 2.16 .

Aqui, vamos dividir a demonstração em partes.

1. Os extremos de cada intervalo restante, após uma etapa, apresentam apenas os algarismos o e 2 em sua representação na base 3: na primeira etapa, os extremos dos intervalos obtidos são escritos como

$$
(0,0,0,0, \cdots 0 \cdots)_{3},(0,1,0,0, \cdots 0 \cdots)_{3},(0,2,0,0, \cdots 0 \cdots)_{3} \text { e }(1,0,0,0, \cdots 0 \cdots)_{3},
$$

ou seja, respectivamente, $0, \frac{1}{3}, \frac{2}{3}$ e 1 .

Na segunda etapa, podemos escrever os novos extremos obtidos como

$$
(0,0,1,0, \cdots 0 \cdots)_{3},(0,0,2,0, \cdots 0 \cdots)_{3},(0,2,1,0, \cdots 0 \cdots)_{3} \text { e }(0,0,2,2, \cdots 0 \cdots)_{3} .
$$

Notemos que na primeira etapa temos como conjunto de valores extremos o seguinte: $C_{1}=$ $\left\{0, \frac{1}{3}, \frac{2}{3}, 1\right\}$. 
Na segunda etapa são somados, a cada termo de $C_{1}$ que represente um extremo esquerdo de um intervalo, o valor $\frac{1}{3^{2}}$ e, depois, o valor $\frac{2}{3^{2}}$, resultando que

$$
C_{2}=\left\{\mathbf{0}, 0+\frac{1}{3^{2}}, 0+\frac{2}{3^{2}}, \frac{\mathbf{1}}{\mathbf{3}}, \frac{\mathbf{2}}{\mathbf{3}}, \frac{2}{3}+\frac{1}{3^{2}}, \frac{2}{3}+\frac{2}{3^{2}}, \mathbf{1}\right\}
$$

é o conjunto dos extremos dos intervalos restantes na segunda etapa.

Do fato de que o número $(0,0,0, \ldots 0,2,2, \ldots, 2)_{3}$ com $n$ algarismos 0 seguidos sempre de algarismos 2, a partir da posição $n+1$, é o número $(0,0, \ldots, 0,1,0 \ldots)_{3}$, onde 1 está na posição $n$ (ver Proposição 2.17), podemos afirmar que até o passo $n=2$, todos os elementos de Cantor obtidos nos extremos dos intervalos têm apenas os algarismos 0 ou 2 na base 3 .

Agora, suponhamos que na etapa $n-1$ todos os extremos de intervalos tenham, como representação na base 3 , apenas os algarismos 0 ou 2 , descartando aqui situações em que temos $(0, *, \ldots, *, 0, \underbrace{2}_{\text {posição } n}, 2, \ldots)$, com os *s sendo 0 ou 2 , pois este número, que é um extremo direito, é igual a $(0, *, \ldots, *, 1,0,0, \ldots)$, com 1 na posição $n-1$. Cada intervalo nessa etapa tem comprimento $\frac{1}{3^{n-1}}$. Para passarmos para a etapa $n$, dividimos cada intervalo em três partes, passando a obter intervalos de comprimento $\frac{1}{3^{n}}$, sendo que de cada um é retirado o terço médio aberto. Portanto, os novos extremos são obtidos somando a extremos esquerdos dos intervalos da etapa $n-1$, os valores $\frac{1}{3^{n}}$ e $\frac{2}{3^{n}}$, ou seja, os números

$$
(0,0, \ldots, \overbrace{1}^{=x_{n}}, 0, \ldots)_{3} \mathrm{e}(0,0, \ldots, 0, \overbrace{2}^{=x_{n}}, 0, \ldots)_{3},
$$

sendo $x_{n}$ como indicado na Definição 2.16.

Logo, como a partir do $n$-ésimo dígito os algarismos são nulos para números que são extremos de intervalos na etapa $n-1$, na etapa $n$, todos os extremos esquerdos dos intervalos continuam a ter como algarismos na representação em base 3 , apenas 0 ou 2, pois os novos extremos esquerdos são obtidos somando-se $\frac{2}{3^{n}}$ a extremos esquerdos de intervalos restantes na etapa $n-1$.

2. Os elementos que são extremos de intervalos restantes, em cada etapa, são elementos de $K$ : por construção, eles são mantidos, já que em cada etapa, apenas o terço médio aberto é retirado dos intervalos ainda restantes.

3. Todos os números que admitem apenas 0 ou 2 na representação na base 3 são elementos de $K$ : vamos dividir este item em duas partes. 
(a) Vamos considerar aqui apenas aqueles elementos $x$ cujos dígitos são 0 ou 2, de forma que o dígito 2 aparece apenas uma quantidade finita de vezes. Consideremos que $x=(0,0, \ldots, \underbrace{2}_{=x_{n_{1}}}, 0, \ldots, 0, \underbrace{2}_{=x_{n_{2}}}, 0, \ldots, 0, \underbrace{2}_{=x_{n_{k}}}, 0, \ldots)$, ou seja, o dígito 2 aparece $k$ vezes. Com $x_{1}=(0,0, \ldots, \underbrace{2}_{=x_{n_{1}}}, 0, \ldots)$, temos que $x_{1}$ é extremo esquerdo, na $n_{1}$-ésima etapa, do intervalo $\left[\frac{2}{3^{n_{1}}}, \frac{1}{3^{n_{1}-1}}\right]$. Com $x_{2}=(0,0, \ldots, \underbrace{2}_{=x_{n_{1}}}, 0, \ldots, 0, \underbrace{2}_{=x_{n_{2}}}, 0, \ldots)$, temos $x_{2}$, na $n_{2}$ ésima etapa, como extremo esquerdo do intervalo $\left[\frac{2}{3^{n_{2}}}, \frac{1}{3^{n_{2}-1}}\right]$. Procedendo dessa forma, chegamos a $x_{k}=x=(0,0, \ldots, \underbrace{2}_{=x_{n_{1}}}, 0, \ldots, 0, \underbrace{2}_{=x_{n_{2}}}, 0, \ldots, 0, \underbrace{2}_{=x_{n_{k}}}, 0, \ldots)$ como sendo $\mathrm{o}$ extremo esquerdo, na $n_{k}$-ésima etapa, do intervalo $\left[\frac{2}{3^{n} k}, \frac{1}{3^{n_{k}-1}}\right]$. Ou seja, obtemos que cada número escrito, na base 3 , com dígitos 0 ou 2 , admitindo uma quantidade finita de dígitos iguais a 2, é um extremo esquerdo de um intervalo em alguma etapa do processo de construção de $K$.

(b) Vejamos agora os casos onde pode surgir uma quantidade infinita de dígitos 2. Podemos escrever $c=\left(a_{1}, a_{2}, a_{3}, a_{4}, a_{5}, \ldots\right)_{3}=\sum_{k=1}^{\infty} \frac{a_{k}}{3^{n}}$, em que $a_{k}=0$ ou $a_{k}=2$, ou seja, tal número é limite de uma sequência das somas parciais, isto é, $c=\lim _{j \rightarrow \infty} \sum_{n=1}^{j} \frac{a_{k}}{3^{n}}$, em que cada soma parcial é um elemento de Cantor, por ser extremo esquerdo de algum intervalo restante em uma dada etapa, conforme visto na parte (a). Como $K$ é fechado, então $c \in K$.

4. Existem infinitos elementos de $K$ que não são extremos dos intervalos: vimos que os números da forma $\left(a_{1}, a_{2}, a_{3}, \ldots\right)_{3}$, em que cada $a_{i}, i \in \mathbb{N}$, é igual a 0 ou a 2 , pertencem ao conjunto de Cantor.

Consideremos o número $(0,2,0,2,0,2, \ldots)$, ou seja, o número da forma

$$
\frac{2}{3^{2}}+\frac{2}{3^{4}}+\frac{2}{3^{6}}+\cdots+\frac{2}{3^{2 n}} \cdots
$$

No caso, temos uma progressão geométrica com termo inicial $\frac{2}{3^{2}}$ e razão $\frac{1}{3^{2}}$. Segue, diretamente, que tal soma infinita é $\frac{1}{4}$, ou seja, este é um elemento do conjunto de Cantor.

Mas, $\frac{1}{4}$ não é extremo de um intervalo, pois não tem uma representação, na base 3 , da forma $(0, *, \ldots, *, 1,0, \ldots)$ ou $(0, *, \ldots, *, 2,0, \ldots)$, com $^{*}$ sendo 0 ou 2.

Consideremos agora o número

$$
\frac{2}{3^{3}}+\frac{2}{3^{6}}+\frac{2}{3^{9}}+\cdots+\frac{2}{3^{3 n}} \cdots
$$


Aqui temos uma progressão geométrica com termo inicial $\frac{2}{3^{3}}$ e razão $\frac{1}{3^{3}}$. Segue diretamente que tal soma infinita resulta no número $\frac{1}{13}$. Analogamente ao caso de $\frac{1}{4}, \frac{1}{13}$ não é extremo de nenhum intervalo.

Procedendo dessa forma, temos para cada $j \in \mathbb{N}$, um número dado por

$$
\frac{2}{3^{j}}+\frac{2}{3^{2 j}}+\frac{2}{3^{3 j}}+\cdots+\frac{2}{3^{n j}} \cdots
$$

como elemento de $K$ que não pertence a um extremo, ou seja, existem infinitos elementos de $K$ que não são extremos de nenhum intervalo obtido em uma etapa dada.

5. Todos os elementos de $K$ admitem, na representação em base 3 , apenas os algarismos 0 ou 2: Reforçamos que a cada etapa, retiramos os terços médios dos intervalos restantes da etapa anterior, sendo estes terços médios intervalos abertos.

No caso, na etapa $n$, podemos escrever

$$
C_{n}=\frac{1}{3} C_{n-1} \cup\left(\frac{1}{3} C_{n-1}+\frac{2}{3^{n}}\right)
$$

sendo $\frac{1}{3} C_{n-1}$ o conjunto formado pela união dos terços iniciais de cada intervalo de $C_{n-1} \mathrm{e}$, $\frac{1}{3} C_{n-1}+\frac{2}{3^{n}}$ o conjunto formado pela união dos terços finais de cada intervalo de $C_{n-1}$. Ou seja, os terços médios foram retirados. De outra maneira, podemos escrever:

$$
\begin{aligned}
C_{n} & =C_{n-1} \backslash\left(\bigcup_{k=0}^{3^{n-1}-1}\left(k \frac{1}{3^{n-1}}+\frac{1}{3^{n}}, k \frac{1}{3^{n-1}}+\frac{2}{3^{n}}\right)\right) \\
& =C_{n-1} \backslash\left(\bigcup_{k=0}^{3^{n-1}-1}\left(\frac{3 k+1}{3^{n}}, \frac{3 k+2}{3^{n}}\right)\right) .
\end{aligned}
$$

O último valor de $k$ na união pode ser obtido notando que devemos ter $\frac{3 k+1}{3^{n}}=1-\frac{2}{3^{n}}$ e $\frac{3 k+2}{3^{n}}=1-\frac{1}{3^{n}}$. Nesta outra maneira de escrever, são explicitados os terços médios retirados na etapa $n$.

Agora, verifiquemos que todos os elementos de $K$ representados na forma $\left(0, a_{1} a_{2} \ldots a_{n-1} \ldots\right)_{3}$ têm apenas 0 ou 2 como algarismos.

(a) No primeiro passo, nós vamos para $C_{1}=\left[0, \frac{1}{3}\right] \cup\left[\frac{2}{3}, 1\right]$, removendo $\left(\frac{1}{3}, \frac{2}{3}\right)$.

(b) Notemos que foram removidos todos os números entre $(0,1)_{3}$ e $(0,2)_{3}$. Por exemplo, $(0,1,1)_{3},(0,1,0,0,0,2)_{3}$, etc. Apesar de $(0.1)_{3}$ restar, ele pode ser escrito como $(0,2,2,2,2, \ldots)_{3}$. Ou seja, todos os números que começam com $(0,1)_{3}$ são retirados, exceto o próprio $(0,1)_{3}$. 
(c) Para cada $n \in \mathbb{N}$, podemos, como visto acima, escrever:

$$
\begin{aligned}
& C_{n}=C_{n-1} \backslash\left(\bigcup_{k=0}^{3^{n-1}-1}\left(\frac{3 k+1}{3^{n}}, \frac{3 k+2}{3^{n}}\right)\right)
\end{aligned}
$$

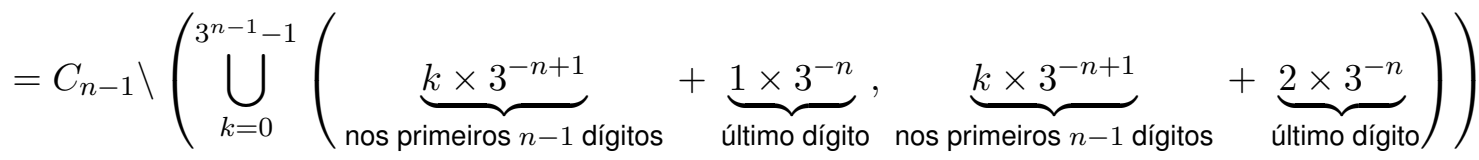

Notemos que todos os números entre $\left(0, a_{1}, a_{2}, \ldots, a_{n-1} 1\right)_{3}$ e $\left(0, a_{1}, a_{2}, \ldots, a_{n-1} 2\right)_{3}$, ou seja, números que começam com $\left(0, a_{1}, a_{2}, \ldots, a_{n-1} 1\right)_{3}$, excetuando-se o próprio número $\left(0, a_{1}, a_{2}, \ldots, a_{n-1} 1\right)_{3}$, não pertencem ao conjunto de Cantor. Um exemplo desses números é o número $\left(0, a_{1}, a_{2}, \ldots, a_{n-1}, 1,0,0,0,2\right)_{3}$.

De fato, acima são retirados os intervalos $\left(\frac{3 k+1}{3^{n}}, \frac{3 k+2}{3^{n}}\right)$, ou seja, todos os intervalos da forma $(x, y)$, em que $x=\left(0, a_{1}, a_{2}, \ldots, a_{n-1}, 1\right)_{3}$ e $y=\left(0, a_{1}, a_{2}, \ldots, a_{n-1}, 2\right)_{3}$, pois

$$
\left\{k 3^{-n+1} ; 0 \leq k \leq 3^{n-1}-1 \mathbf{e} k \in \mathbb{Z}\right\}=\left\{\left(0, a_{1}, \ldots, a_{n-1}\right)_{3} ; a_{1}, \ldots, a_{n-1} \in\{0,1,2\}\right\},
$$

pela Proposição 2.18 .

Como $\left(0, a_{1}, a_{2}, \ldots, a_{n-1} 1\right)_{3}=\left(0, a_{1}, a_{2}, \ldots, a_{n-1}, 2,2,2,2,2 \ldots\right)_{3}$, temos tal número representado com algarismos 0 ou 2.

(d) Segue, assim, que $K$ não contém nenhum número que tenha 1 como algum dígito, concluindo que todos os números de $K$ na base 3 têm como algarismos apenas 0 ou 2 .

\section{Agora provaremos que, de fato, o conjunto de Cantor não é enumerável.}

Seja $S$ o conjunto de todas as sequências infinitas formadas com os termos 0 e 2 . Por exemplo, são elementos de $S$ as sequências $(0,0,0,0,0,0, \ldots),(0,2,0,2,0,2, \ldots)$ e $(2,0,2,0,2,0, \ldots)$. Vamos mostrar que nenhum subconjunto enumerável $L=\left\{s_{1}, s_{2}, \ldots, s_{n}, \ldots\right\}$ de $S$ é igual a $S$, ou seja, $S$ não é enumerável.

Denominemos $s_{n n}$ o $n$-ésimo termo de $\left(s_{n}\right)$. Seja $s * \in S$ construída da seguinte maneira: se $s_{n n}=0$, então o $n$-ésimo termo de $s *$ é 2 e, se $s_{n n}=2$, então o $n$-ésimo termo de $s *$ é 0 . Isto é ilustrado a seguir, através de um exemplo. 
Figura 2 - Diagonal de Cantor.

$$
\begin{array}{rllllllllll}
s_{1} & = & \mathbf{0} & 0 & 0 & 0 & 0 & 0 & 0 & 0 & \ldots \\
s_{2} & = & 2 & \mathbf{0} & 2 & 0 & 2 & 0 & 2 & 0 & \ldots \\
s_{3} & = & 0 & 2 & \mathbf{2} & 2 & 0 & 0 & 2 & 2 & \ldots \\
s_{4} & = & 2 & 2 & 0 & \mathbf{0} & 2 & 2 & 0 & 0 & \ldots \\
s_{5} & = & 0 & 0 & 0 & 2 & \mathbf{2} & 0 & 0 & 0 & \ldots \\
s_{6} & = & 2 & 0 & 2 & 0 & 2 & \mathbf{0} & 2 & 0 & \ldots \\
s_{7} & = & 0 & 2 & 0 & 2 & 0 & 2 & \mathbf{0} & 2 & \ldots \\
s_{8} & = & 2 & 2 & 2 & 2 & 0 & 2 & 2 & \mathbf{2} & \ldots \\
\vdots & \vdots & \vdots & \vdots & \vdots & \vdots & \vdots & \vdots & \vdots & \vdots & \ddots \\
s^{*} & = & 2 & 2 & 0 & 2 & 0 & 2 & 2 & 0 & \ldots
\end{array}
$$

Fonte: Elaboração dos autores (2020).

Construímos uma sequência $s *$ diferente de todas as do conjunto $L$.

Temos uma bijeção $K \longrightarrow S$ dada por $\left(0, a_{1}, a_{2}, \ldots\right)_{3} \longmapsto\left(a_{1}, a_{2}, \ldots\right)$, com $a_{i}$ sendo 0 ou 2 para todo $i \in \mathbb{N}$. Logo, de $S$ não ser enumerável, segue diretamente que $K$ não é enumerável.

\section{Uma propriedade adicional do Conjunto de Cantor}

Definição 4.1. Dado um intervalo $I=[a, b]$, o comprimento do intervalo $I$ é dado por $b-a$.

Teorema 4.2. O Conjunto de Cantor tem comprimento nulo.

Prova. Na construção do conjunto de Cantor $K$ é retirado, primeiramente, do intervalo [0,1], um intervalo de comprimento $\frac{1}{3}$, depois dois intervalos de comprimento $\frac{1}{3^{2}}$, na sequência quatro intervalos de comprimento $\frac{1}{3^{3}}$ e, assim por diante. Ou seja, a cada etapa $n$, são retirados $2^{n-1}$ intervalos de comprimento $\frac{1}{3^{n}}$. Logo, podemos dizer que o comprimento de $K$ é

$$
1-\sum_{n=1}^{\infty} \frac{2^{n-1}}{3^{n}}=1-\frac{1}{3} \sum_{n=1}^{\infty}\left(\frac{2}{3}\right)^{n-1}=1-\frac{1}{3} \sum_{n=0}^{\infty}\left(\frac{2}{3}\right)^{n}=1-\frac{1}{3} \frac{1}{1-\frac{2}{3}}=0
$$




\section{Duas propriedades topológicas}

Nesta seção apenas enunciaremos duas propriedades importantes relativas ao conjunto de Cantor, cujas demonstrações podem ser encontradas em Willard (2004, Corolário 30.4 e Teorema 30.7). As ferramentas não serão apresentadas aqui, por serem longas e não fazerem parte do objetivo principal deste texto.

Teorema 5.1. (Teorema de Brouwer) Todo espaço métrico não vazio, compacto, perfeito e totalmente desconexo é homeomorfo ao conjunto de Cantor.

Prova. Ver Willard (2004, Corolário 30.4).

Teorema 5.2. Todo espaço métrico compacto $X$ é uma imagem contínua do conjunto de Cantor $K$, ou seja, dado o espaço métrico compacto $X$, existe uma função contínua $f: K \longrightarrow X$ tal que

$$
f(K)=X
$$

Prova. Ver Willard (2004, Teorema 30.7).

\section{Comentários finais}

O conjunto de Cantor é um tanto curioso, pois não é esperado que conjuntos satisfaçam, simultaneamente, as quatro propriedades listadas na Seção 3. Além dessas propriedades, ainda temos as elegantes propriedades topológicas listadas na seção anterior. Vimos ainda o fato de o Conjunto de Cantor ter medida nula. Além disso, os espaços topológicos homeomorfos ao conjunto de Cantor são chamados Espaços de Cantor.

\section{Referências}

BROUWER, L. E. J. On the structure of perfect sets of points. KNAW, Proceedings, v. 23, n. 4, p. 397-399, 1910.

FREIRA, A. A. A teoria dos conjuntos de cantor. Paidéia, Ribeirão Preto, n. 2, fev./jul. 1992. DOI: https://doi.org/10.1590/S0103-863X1992000200008.

IMPA. Instituto de Matemática Pura e Aplicada. Georg Cantor (1845-1918) - pai do infinito e do ICM. 15 dez. 2017. Disponível em:

https://impa.br/noticias/georg-cantor-1845-1918-pai-do-infinito-e-do-icm/. Acesso em: 06 jun. 2020. 
LIMA, E. L. Análise Real. v. 1, 12. ed. Rio de Janeiro: IMPA, 2018.

LIMA, E. L. Curso de Análise. v. 2, 11. ed. Rio de Janeiro: IMPA, 2014.

MATHONLINE. Compact Sets in a Metric Space are Closed and Bounded. Disponível em:

http:

//mathonline.wikidot.com/compact-sets-in-a-metric-space-are-closed-and-bounded Acesso em: 23 jun. 2020.

MUNKRES, J. R. Topology. 2. ed. New Jersey: Prentice Hall, 2000.

WILLARD, S. General Topology. Mineola N. Y.: Dover Publications, 2004. 